\title{
Cistinuria y Litiasis Renal
}

\author{
Dra. M. Eugenia Willshaw $Z^{1}{ }^{1}$; Q.F. Eliana Gatindo F. $^{2}$; Q.F. Alfonso Salinas T. ${ }^{3}$; \\ Dr. Raimundo Ariztía $\mathbf{M}^{+}$
}

\section{Cystinuria and Urolithiasis}

The case of a 9 years old girl with bilateral nephrolithiasis, in whom cystinuria (probably tipe I) was demostrated, is presented. The same metabolic abnormality was detected in her monozigote twin sister, who had been considered normal. A pyelographic study disclosed a silent renal lithiasis in this girl. Both parents showed decreased intestinal absortion for the aminoacid when challenged with oral administration of cystine. To our knowledge no other cases of cystinuria in twins have been communicated in the literature.

La cistinuria es un defecto hereditario en el transporte de aminoácidos dibásicos en las células epiteliales de los túbulos renales y el tracto gastrointestinal ${ }^{1}$.

Se expresa clinicamente por la formación de cálculos urinarios al precipitar la cistina, el menos soluble de los aminoácidos dibásicos excretados

1 Médico Endocrinólogo Infan til. Hospital Militar.

2 Químico Farmacéutico. Laboratorio Clínico. Hospital Militar.

3 Quínico Farmacéutico. Fac. de Medicina Sur. Universidad de Chile.

4 Médico. Jefe Servicio Lrologia. Hospital Militar. en exceso. Los otros son la lisina, la arginina y la ornitina.

De las causas de litiasis renal, la cistinuria es la que representa el menor porcentaje $(2,9 \%)^{2}$.

$\mathrm{La}$ incidencia es difícil de precisar, ya que el defecto genético puede presentarse en forma completa o incompleta. Si se consideran sólo a los homozigotos, ella puede estimarse en $1 / 100.000$ individuos.

La primera descripción de cistinuxia fue hecha por Wollaston ${ }^{3}$ quién en 1810 analizó dos cálculos vesicales diferentes a los conocidos hasta ese momento. En 1824 , Stromeyer ${ }^{4}$ señaló la presen- 
cia de cristates hexagonales en la orina de pacientes cistinúricos.

En 1833, Berzelius ${ }^{5}$ denominó cistina a este compuesto formador de cálculos, y Friedman en $1902^{6}$ definió su estructura química.

Dent y Rose $^{7}$ en 1952, considerando la similitud estructural de la cistina con los otros aminoácidos dibásicos antes mencionados, postularon la existencia de un mecanismo único alterado o ausente en el rifón de estos enfermos. En 1962, Milne $^{a}$ demostró el defecto intestinal concomitante que compromete la absorción intestinal de la cistina junto con la de los otros aminoaicidos dibásicos.

Por lo tanto, esta sería la primera enfermedad metabólica descrita que compromete a un sistema de transporte común a varios aminoácidos de similar estructura quimica ${ }^{5}$.

\section{Caso Clínico}

Paciente de 9 años, sexo femenino, controlada en el Servicio de Urología đel Hospital Militar de Santiago, por infección urinaria prolongada y litiasis renal bilateral. Por encontrarse una calcemia elevada $(14,0 \mathrm{mg} / \mathrm{dl})$ se solicitó su estudio en Endocrinología Infantil. Peso $28 \mathrm{~kg}$. talla 1,29 m. (percentil 25 para edad cronológica); frecuencia cardíaca 70 par minuto, regular; presión arterial: $110 / 60 \mathrm{~mm}$ de $\mathrm{Hg}$. Sin signos de desarrollo puberal. En el abdomen se palpa polo inferior del riñón derecho. Orina: densidad 1.010; reacción alcalina; proteinuria leve; piocitos: 20 por campo; eritrocitos: 0-2 por campo. En el urocultivo se desarrollaron incontables colonias de Klebsiella y E, coli. Nitrógeno ureico en sangre: 15 $\mathrm{mg} / \mathrm{dl}$; Uricemia: $3,5 \mathrm{mg} / \mathrm{d} 1$. $\mathrm{pH}$ en sangre 7,41 ; CO 2 total: $25,1 \mathrm{mEq} / 1$. Electrolitos plasmáticos: Sodjo: $143 \mathrm{mEq} / 1$; Potasio: $4,1 \mathrm{mEq} / 1$; Cloruros: $103 \mathrm{mEq} / \mathrm{l}$. Después de la Calcemia inicial $\mathrm{de} 14,0 \mathrm{mg} / \mathrm{dl}$, en dos ocasiones más se encontraron concentraciones de $10,5 \mathrm{mg} / \mathrm{dl}$. La Tabla 1 muestra los resultados de determinaciones de Calcio y Fósforo en distintas condiciones. Proteinemia, electroforésis de proteinas plasmáticas y tiempo de Protrombina eran normales.

En la orina se encontraron cristales de cistina después de acidificar la muestra y la reacción con Nitroprusiato fue positiva. También se encontró aminoaciduria por eliminación excesiva de Cistina y Lisina.

El coeficiente intelectual de la paciente resultó normal.

Las radiografías de cráneo, hombro, mano y dientes tambièn fueron normales.

Su hermana gemela, considerada sana hasta el momento, tenía una pequeña imagen de litiasis renal derecha, cistinuria y lisinuria aumentadas; una serie de cinco calcemias dio como resultado un promedio de $9,25 \mathrm{mg} / \mathrm{d} 1$ con una desviación standard de $0,34 \mathrm{mg} / \mathrm{dl}$.

Ni el padre, ni la madre, ni el resto de los hermanos de la paciente eliminaban Cistina sobre los valores considerados normales (Cromatografía normal, reacción de nitroprusiato negativa).

En la paciente, su gemela y sus padres se dio una sobrecarga oral de cistina en dosis de 0,05 micromoles por kilo de peso corporal ${ }^{9}$, con los

Tabla 1.

Calcemias, Fosfemias y Calciurias en Distintas Circunstancias

\begin{tabular}{|c|c|c|c|c|c|}
\hline & $\begin{array}{l}\text { Calcemia } \\
\mathrm{mg} / \mathrm{dl}\end{array}$ & $\begin{array}{l}\text { Fosfemia } \\
\text { mg/d } 1\end{array}$ & $\mathrm{mg} / 24 \mathrm{hs}$. & $\begin{array}{c}\text { Calciuria } \\
\mathrm{mg} / \mathrm{kg} \text { peso }\end{array}$ & $\mathrm{mg} / \mathrm{g}$ Creatinina \\
\hline $\begin{array}{l}\text { Régimen normal de } \\
\text { Calcio }\end{array}$ & $10,2 \pm 1,8$ & $4,3 \pm 0,1$ & 150 & 5,0 & - \\
\hline Régimen hipocálcico & 9,5 & & 106 & 3,5 & 94 \\
\hline $\begin{array}{l}\text { Con sobrecarga de } \\
\text { calcio }\end{array}$ & 10,3 & & 294 & 9,450 & \\
\hline $\begin{array}{l}\text { Prueba de sobrecarga } \\
\text { oral con } 1 \mathrm{~g} \text { de calcio } \\
\text { (Pak): } \\
\text { Basal }\end{array}$ & 9,5 & & 21,6 & & $0,09^{1}$ \\
\hline Post ingesta & 10,3 & & $\begin{array}{c}49,0 \\
(2 \text { horas })\end{array}$ & & $0,45^{2}$ \\
\hline
\end{tabular}

1 Normales: menos de 0,11

2 Normales: menos de 0,20

3 Media y desviación standard de 10 determinaciones en el caso de calcemia y fosfenta. 
resultados que se muestran en la Tabla 2, de los que se puede concluir que en los cuatro, las concentraciones de cistisa plasmática se mantuvieron sin alza significativa durante las dos horas siguientes a su administracion oral (Fig. 1). La concentración de cistina en todas las muestras corresponde a los valores normales en niños (0,55-0,92 $\mathrm{mg} / \mathrm{d} 1)$ o adultos $(0,58-1,70 \mathrm{mg} / \mathrm{d}])$ en ayunas. Los sujetos nomales tienen, después de la administración dosis similares, curvas de concentración plasmática que aumentan hasta una concentración máxima al cabo de 1 hora, manteniéndose significativamente sobre el valor basal hasta tres horas despues de la sobrecarga ${ }^{9}$. Los homozigotos tipo I y II muestran curvas planas como las mostradas por estos pacientes después de la sobrecarga con el aminoácido ${ }^{9}$. Los heterozigotos del tipo III presentan una absorción retardada; que en los casos que el presenta, tiene un máximo a las dos horas ${ }^{9}$ (Fig. 2).

Tabla 2 .

Concentratraciones plasmáticas de cistina desptés de sobrecarga con el aminoácido $(0,05$ umoles/ $\mathrm{kg}$ peso):

La concentración apatece expresada en $\mathrm{mg} / \mathrm{dl}$.

$\begin{array}{lcccc} & \text { Paciente } & \text { Gemela } & \text { Padre } & \text { Madre } \\ \text { Basal } & 0,93 & 1,45 & 0,85 & 0,92 \\ 30 \text { minutos } & 1,10 & 1,65 & 0,90 & 0,95 \\ 2 \text { horas } & 1,00 & 1,43 & 0,90 & 0,95 \\ \text { V. Ref: Niños menores de } 10 & \text { años: } 0,55-0,92 \\ \text { mg/d1 adultos } 0,58-1,70 \mathrm{mg} / \mathrm{d} 1 .\end{array}$

PRUEBA DE SOBRECARGA ORAL. DE CISTINA (D $=0.05 \mu$ moles $/ \mathrm{kg}$.)

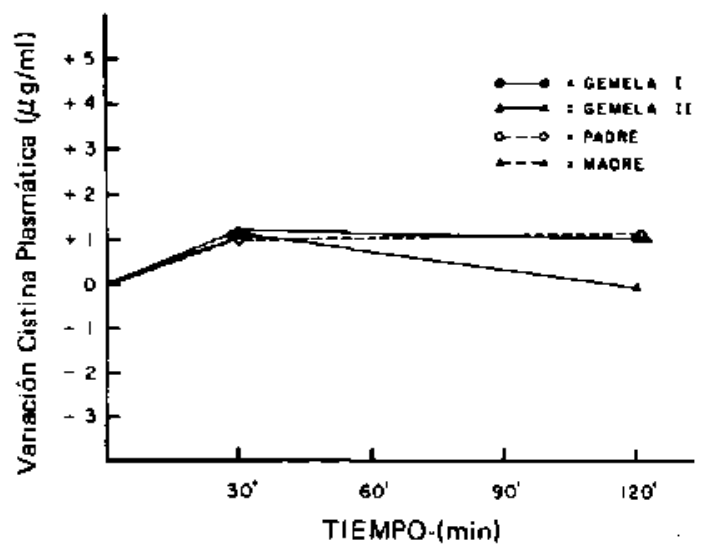

Figura 1.: Curva de variación de cistina plasmática antes $y$ luego de una sobrecarga oral de cistina a la paciente, sus padres y su gemela.

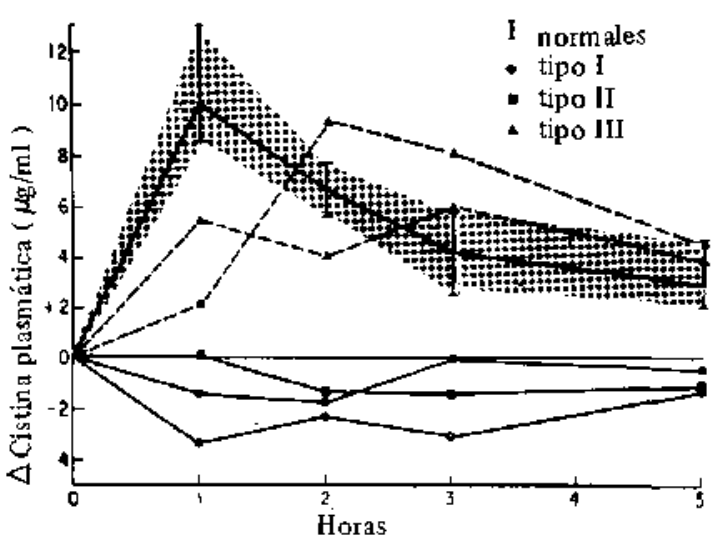

Figura 2.: Clasificación de cistinuria según Stanbury ${ }^{9}$, de acuerdo a curva de variación plasmática de niveles de cistina luege de sobre carga oral.

En nuestros pacientes no hubo cambios significativos ni media hora ni dos horas después de la sobrecarga. No nos pareció necesario prolongar más tiempo los controles porque, aún cuando los casos de Stambury ${ }^{9}$ muestran un máximo a las dos horas y en nuestros pacientes pudiera darse uno todavía más tardio, los casos presentados por dicho autor, mostraban a la hora diferencia significativa entre los heterozigotos I y II y los del tipo III, que se hacía más intensa a las dos horas.

El estudio de los dermatoglifos de ambos pacientes, padres y hermanos no mostró ningún patrón característico.

El genotjpo sanguíneo de las gemelas fue o-IV Rh(+), heterozigoto para el factor D.

\section{COMENTARIO}

Presentamos una niña de 9 años de edad, portadora de una infección urinaria y litiasis renal, que no ha expulsado cálculos que permitan su análisis químico hasta la actualidad.

Los exámenes de laboratorio demuestran en ella y su hermana gemela, un trastorno metabóljco de la cistina, con eliminación aumentada del aminoácido en la orina (nitroprusiato positiva, cromatografía que muestra cistina y lisina au. mentadas).

En el padre, madre y hermanos no se demostró eliminación aumentada de cistina en la orina.

Al someter a la paciente y su gemela así como a sus padres a una sobrecarga oral de cistina, se observaron niveles plasmáticos que no experimentaron un alza significativa hasta dos horas después de la administración del aminoácido. Esto demuestra un defecto en la absorción del aminoácido.

El comportamiento frente a la sobrecarga oral 
de cistina y los hallazgos diferentes en la orina de la paciente y su gemela respecto a sus padres y otros hermanos, permiten postular una cistinuria de tipo I ó II, considerando a la paciente y su gemela como probable homozigotos y a sus padres como heterozigotos (Tabla 3).

Las calcemjas altas presentadas por la proban. do y su gemela, con nomnalidad posterior por lo menos en siete oportunidades, unidas a hiperabsorción intestinal de una sobrecarga de $1 \mathrm{~g}$. de Calcio oral (según Pak), pueden ser interpretadas como secundarias a administración masiva y repetida de Calcio y Vitamina D que recibieron por indicación de sus padres y sin prescripción médica.

Este hecho, al igual que el publicado en la literatura ${ }^{10}$ predispone a la formación de cálculos en un sujeto que elinina cistina en cantidades aumentadas en la orina.

Conviene prevenir a los individuos cistinúricos respecto a la ingestión de cantidades excesivas de Calcio en los alimentos y a aportes suplementarios de Vitamina D, para no contribuir a la formación de cálculos urinarios. Asimismo, al coexistir ambas litiasis (cistinúrica e hipercalciúrica) deben adoptarse medidas que no empeoren esta condición (Ej. modificación del $\mathrm{pH}$ de la orina, ya que al alcalinizar precipita el calcio, $y$ al acidificar, precipita la cistina). Es por esto que en nuestra paciente y su gemela indicamos un aporte de liquido superior a dos litros diarios, aportes mínimos de Calcio, tratamiento de la infección urinaria y mantención de $\mathrm{pH}$ urinario.

El uso de drogas antiquelantes como penicilamina parece justificado sólo en casos de recidivas o riesgos que superen a aquellos derivados de la droga misma 3 .

Nuestras pacientes tienen un cuociente intelectual normal y buen rendimiento escolar, hecho que se evaluó especialmente en consideración a que la cistinuria puede asociarse con retardo mental $y$ porque su frecuencia es diez veces mayor en poblaciones de retardados mentales que en la población general ${ }^{12}$.

En resumen, hemos demostrado en una paciente de 9 años, la existença de un trastorno metabólico que involucra a la cistina y que reconoce base genética y una alteración similar en su hermana gemela, considerada sana hasta antes de someterla al estudio.

Mediante la respuesta a sobrecarga oral de cistina en las gemelas y sus padres se pudieron clasificar tentativamente en el tipo I, aceptando que sus padres sean heterozigotos y que la expresión completa se produzca exclusivamente en los homozigotos. Este defecto intestinal heredado tiene poca significación clínjca pero constituye un buen marcador genético y ha permitido una nueva clasificación de esta enfermedad.

Nos pareció de interés comunicar este caso, pues no se encuentran publicaciones intemacionales sobre esta afección en gemelos.

\section{AGRADECIMIENTOS}

Al Dr. Hugo Pumarino, Jefe del Departamento de Endocrinología, Facultad de Medicina Norte, Universidad de Chile. Hosp. José Joaquín Aguirre.

Al Dr. manuel Santos A. Departamen to de Biologia Celular. Facultad de Ciencias Biológicas. Pontificia Universidad Católica de Chile.

Tabla 3.

Clasifúcación de casos de Cistinuria(*)

\begin{tabular}{|c|c|c|c|}
\hline $\begin{array}{l}\text { Observaciones } \\
\text { Experimentales }\end{array}$ & Tipo I & Tipo II & Tipo III \\
\hline Recesividad & Completa & Incompleta & Incompleta \\
\hline $\begin{array}{l}\text { Homozigotos: } \\
\text { Transporte "in vitro" } \\
\text { en biopsias de yeyuno }\end{array}$ & $\begin{array}{l}\text { No hay transporte } \\
\text { de cistina, lisina } \\
\text { o arginina }\end{array}$ & $\begin{array}{l}\text { No hay transporte } \\
\text { de lisina }\end{array}$ & $\begin{array}{l}\text { Transporte de cistina } \\
\text { normal o reducido. } \\
\text { Transporte de lisina } \\
\text { reducido en forma } \\
\text { variable. }\end{array}$ \\
\hline $\begin{array}{l}\text { Administración oral } \\
\text { de cistina }\end{array}$ & $\begin{array}{l}\text { No hay elevación } \\
\text { plasmática de } \\
\text { cistina }\end{array}$ & $\begin{array}{l}\text { No hay elevación } \\
\text { de cistina }\end{array}$ & $\begin{array}{l}\text { Elevación de cistina } \\
\text { plasmática }\end{array}$ \\
\hline $\begin{array}{l}\text { Heterozigotos: } \\
\text { Excreción de } \\
\text { aminoácidos } \\
\text { urinarios. }\end{array}$ & Normal & $\begin{array}{l}\text { Cistina y lisina } \\
\text { scbre lo nomnal }\end{array}$ & $\begin{array}{l}\text { Cistina y lisina } \\
\text { sobre lo normal. }\end{array}$ \\
\hline
\end{tabular}

(*) Reproducido en parte de Thiet y Segal ${ }^{3}$ 


\section{REFERENCIAS}

1 Caan, A,G.: Cystinuria: the disease and its models. Life Sc. 28: 5, 1981.

2 Pruzansky, W.: Cystinuria and Cystinuric Urolitiasis in Childhood. Acta Paed. Scand. 55: 97, 1966.

3 Wollaston, W.H.: Phil. Trans. Roy. Soc. London. 100: 223, 1810 .

4 Stromeyer, J.M,: Citado en "The metabolic basis of inherited disease: Stambury, J.B., editor Mc. Graw Hill, New York, 1972.

s Berzelius, J.J.: Calculus urinaires. Traite de Chemic. 7: 424, 1833

${ }^{6}$ Fiedman, R.: Neitr. Chem. Physiol. Pathol. 3: 1, 1902.
7 Dent. C.E. and Rose, G.A.: Aminoacid metabolism in cystinuria. Quatt. J. Med. 20: 20S, 1951.

- Milne, M.D.,; Asatoor, A.M.; Edwards, K.D. and Loughridge, $L, W$ : The in testinal absortion defect in cystinuria, Gut 2: 323, 1961.

9 Stanbury, J.B.; Wyngadardner, J.B.; Frederickson, $D, S_{1}$ : The metabolic basis of inherited disease. Mac Grow Hill, New York, 1972.

10 Dahlberg, P.; Van der Berg, C.: Clinical Features and management of cystinuria. Mayo Clin. Proc. 52:533. 1977.

11 O'Reagan, S.; Robitaille, P.; Mongeau, J.G. and Homsy, $Y_{\text {.: }}$ Cystine calcium bladder calculus in two years old child. J. Urol. 123: 77, 1980.

12 Kurczynski, T.W.. Neurologieal complicatioms of cystinuria. Dev, Med. Child. Neurot. 21: $811,1979$. 